\title{
UHF-Dielectrophoresis Crossover Frequency as a New Marker for Discrimination of Glioblastoma Undifferentiated Cells
}

\author{
Rémi Manczak ${ }^{\circledR}$, Sofiane Saada, Thomas Provent, Claire Dalmay, Barbara Bessette, Gaëlle Bégaud, Serge Battu, \\ Pierre Blondy, Marie-Odile Jauberteau, Canan Baristiran Kaynak ${ }^{\circledR}$, Mehmet Kaynak, Cristiano Palego, \\ Fabrice Lalloué, and Arnaud Pothier
}

\begin{abstract}
This paper introduces the first results of dielectric spectroscopy characterization of glioblastoma cells, measuring their crossover frequencies in the ultra-high-frequency range (above $50 \mathrm{MHz}$ ) by dielectrophoresis (DEP) techniques. Experiments were performed on two glioblastoma lines U87-MG and LN18 that were cultured following different conditions, in order to achieve different phenotypic profiles. We demonstrate here that the presented DEP electrokinetic method can be used to discriminate the undifferentiated from the differentiated cells. In this study, microfluidic lab-on-chip systems implemented on bipolarcomplementary oxide semiconductor technology are used allowing single cell handling and analysis. Based on the characterizations of their own intracellular features, both the selected glioblastoma (GBM) cell lines cultured in distinct culture conditions have shown clear differences of DEP crossover frequency signatures compared to the differentiated cells cultured in a normal medium. These results support the concept and validate the efficiency for cell characterization in glioblastoma pathology.
\end{abstract}

Index Terms-BiCMOS chip, biological cell manipulation, glioblastoma cells, high frequency dielectrophoresis.

$\mathbf{G}$

\section{INTRODUCTION} 240,000 brain tumor new cases were diagnosed worldwide; the majority are GBMs with an incidence of 3-4 per 100 000 persons per year [1]. Conventional therapeutic strategy is mainly surgery, in combination with chemo- and radiotherapy

Manuscript received September 3, 2018; revised November 29, 2018; accepted January 9, 2019. Date of publication; date of current version. This work was supported by the European Union's Horizon 2020 Research and Innovation Program under Grant 737164. (Corresponding author: Remi Manczak.)

R. Manczak, T. Provent, C. Dalmay, P. Blondy, and A. Pothier are with XLIMUMR 7252, University of Limoges/CNRS, Limoges 87060, France (e-mail: remi.manczak@xlim.fr; thomas.provent@xlim.fr; claire.dalmay@xlim.fr; pierre.blondy@xlim.fr; arnaud.pothier@xlim.fr).

S. Saada, B. Bessette, G. Bégaud, S. Battu, M. O. Jauberteau, and F. Lalloué are with CAPTuR-EA 3842, University of Limoges, Limoges 87025 , France (e-mail: sofiane.saada@unilim.fr; barbara.bessette@unilim.fr; gaelle. begaud@unilim.fr; serge.battu@unilim.fr; m-o.jauberteau-marchan@unilim.fr; fabrice.lalloue@unilim.fr).

C. Baristiran Kaynak and M. Kaynak are with IHP, Frankfurt 15236, Germany (e-mail: baristiran@ihp-microelectronics.com; kaynak@ ihp-microelectronics.com).

C. Palego is with Bangor University, Bangor LL57 1UT, U.K (e-mail: c.palego@bangor.ac.uk).

Digital Object Identifier 10.1109/JERM.2019.2895539 according to Stupp protocol. Despite recent advances in surgery, imaging, radiation therapies and chemotherapy, the median survival is less than 15 months [2]. This dark prognosis of GBM is primarily due to the recurrence of tumor, which is resistant to pre-cited conventional treatments [1].

Limited advances in glioblastoma treatment are closely linked to the existence of a restricted cell subpopulation also called cancer stem cells (CSCs), some very immature and undifferentiated cells, responsible for tumor cell heterogeneity [3]. However, even genetically diverse clones express undifferentiated cell markers related to cancer stem cells such as CD133 and CD44. The higher expression levels of CD133 have been correlated to poorer prognosis suggesting that this marker might play a significant role in the resistance of this type of cancer to chemotherapy and radiotherapy [4]. Other markers such as the transcription factors OCT-4, SOX2, pSTAT3, and NANOG are considered as key players in regulating transcription of glioblastoma CSCs [3]. These CSCs are a subpopulation of undifferentiated cells, which have specific biological properties similar to normal stem cells. Currently, biologists use some immunostaining approaches to characterize CSC populations, as flow cytometry, optical microscopy or protein array analysis, based on targeting a set of undifferentiated markers previously described. These markers are required to validate the stemness lineage of CSCs from the huge heterogeneous cell population. Nevertheless, CSCs subpopulation are very rare in tumors and their isolation often requires enriching them in specific culture medium. This strategy is time consuming and delays the results. Currently, the key objective is to try to get around this problem by establishing a new way to discriminate and sort undifferentiated cell populations specifically according to theirs biological and physical characteristics.

To optimize the diagnosis and prognosis methods, the development of different approaches and techniques based on bioelectric signals of cells have been proved to carry various helpful information on cell status [5]. Many sources of cell bioelectric signals, like sodium potassium channels and pumps in the plasma membrane, may affect chemical analytes homeostasis, cell patterning and cell-to-cell interactions with the extracellular matrix, which can be determined by exploiting the dielectric properties. Among these techniques, Dielectrophoresis (DEP) is a label-free, accurate, fast, and low-cost analysis method that 


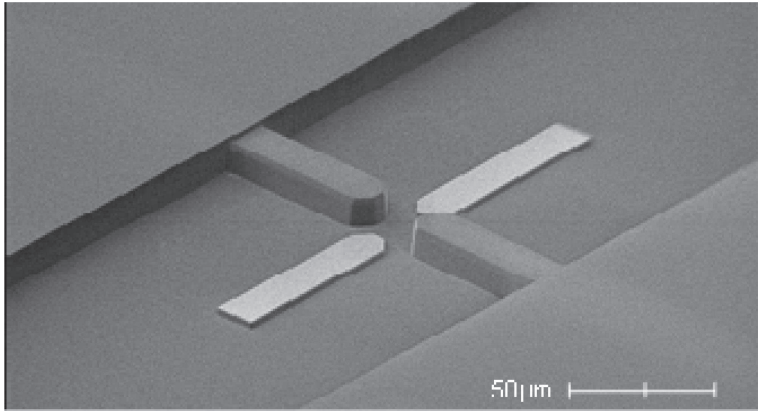

Fig. 1. Quadrupole electrodes system implemented in BiCMOS back end of line SG25H4 technology from IHP.

111 force can be then computed using equation (1).

$$
\begin{aligned}
F_{D E P} & =2 \pi \varepsilon_{m} r^{3} \operatorname{Re}[K(\omega)] \nabla\left|E_{r m s}\right|^{2} \\
K(\omega) & =\left(\frac{\varepsilon_{p}^{*}-\varepsilon_{m}^{*}}{\varepsilon_{p}^{*}+2 \varepsilon_{m}^{*}}\right) \\
\varepsilon_{x}^{*} & =\varepsilon_{0} \varepsilon_{x}-j \frac{\sigma_{x}}{\omega}
\end{aligned}
$$

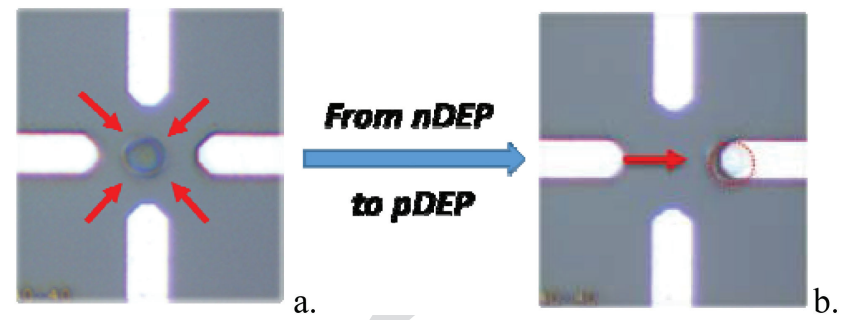

Fig. 2. DEP single cell manipulation principle. (a) Cell repulsion at system center with nDEP. (b) Cell attraction toward electrode with pDEP.

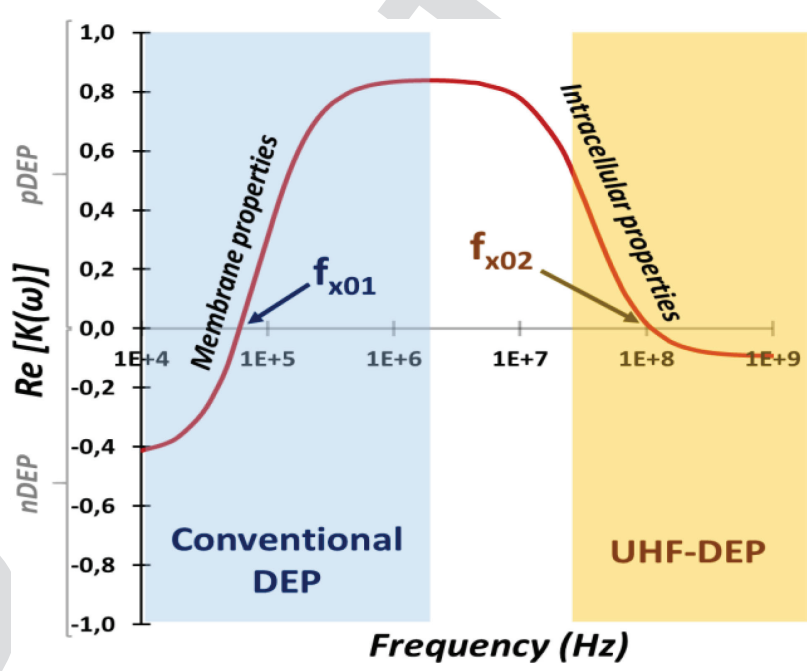

Fig. 3. Typical DEP spectral signature of cell with its two crossover frequencies $\mathrm{f}_{\mathrm{xo} 1}$ and $\mathrm{f}_{\mathrm{xo} 2}$, respectively, at low and high frequency regimes.

where $r$ is the particle radius, $\omega$ is the angular frequency of the 112 applied electric field, $E_{r m s}$ is the root mean square value of this 113 electric field, $\nabla$ is the gradient operator and $\operatorname{Re}[K(\omega)]$ the real 114 part of Claussius-Mossotti factor $K(\omega)$ given by (2) in which $\varepsilon_{p} * 115$ and $\varepsilon_{m} *$ refer to the complex permittivity of the particle and the 116 suspension medium, respectively. The complex permittivity $\varepsilon_{x} * 117$ are defined in (3), where $\varepsilon_{x}$ and $\sigma_{x}$ are the relative permittivity 118 and conductivity either of particles or immersion medium, and 119 $\varepsilon_{0}$ represent the electric constant $\left(8.85410^{-12} \mathrm{~F} \mathrm{~m}^{-1}\right) . \quad 120$

By changing the frequency of the applied electric field [13], 121 the polarized particles would behave in various ways depending 122 on the magnitude and the sign of $\operatorname{Re}[K(\omega)]$ which is in turn 123 determined by the effective conductivity and permittivity of the 124 particle and the dielectric properties of the surrounding medium. 125 Therefore, particles can be individually electro-manipulated ac- 126 cording to their own dielectric properties.

Actually, the generated force is repulsive when $\operatorname{Re}[K(\omega)]$ is 128 negative, meaning that the particle is repealed away from elec- 129 trodes (see Fig. 2(a) negative DEP case - nDEP). Whereas when 130 $\operatorname{Re}[K(\omega)]$ is positive, the force is attractive and the particle 131 moves toward the electrodes where the electric field magnitude 132 is high (see Fig. 2(b) positive DEP case - pDEP). When the 133 force becomes null just before the cell switches to negative to 134 positive DEP (or vice versa), the DEP crossover frequency is 135 then reached. This frequency can be thus considered as charac- 136 teristic of a cell own properties and specificities and may differ 137 between different cells. 
139 Depending on the type of cell properties one wants to access, 140 the choice of the DEP frequency range is important [17]-[19]. 141 If information about cell plasma membrane specificities are 142 sought, conventional DEP frequencies (typically from $100 \mathrm{kHz}$ 143 to $5 \mathrm{MHz}$ ) are very suitable for cell analysis. At this low fre144 quency range, the cell shape, morphology and size have strong 145 influence on the interaction with the electric field. Conversely, 146 Ultra High Frequencies DEP (from $50 \mathrm{MHz}$ to $500 \mathrm{MHz}$ ) will 147 be better to provide information about intracellular properties. 148 Indeed, when frequency increases above several tens of $\mathrm{MHz}$, 149 the plasma membrane lets the electric field penetrate the cell 150 and interact directly with the cell interior. As a result, the effect 151 of DEP forces generated (i.e., attractive vs. repulsive) at high 152 frequency regimes may be different according to the dielectric 153 properties of the cell content. This UHF-DEP characteristic is 154 often presented in the literature [7], [20], [21] and the crossover 155 frequency $f_{x o 2}$ can be written by

$$
f_{x 02}^{2}=\frac{1}{4 \pi^{2}} \frac{1}{\varepsilon_{0}^{2}} \frac{\left(\sigma_{m}-\sigma_{p}\right)\left(\sigma_{p}+2 \sigma_{m}\right)}{\left(\varepsilon_{p}-\varepsilon_{m}\right)\left(\varepsilon_{p}+2 \varepsilon_{m}\right)} .
$$

For an aqueous solution, considering $\varepsilon_{m}>\varepsilon_{p}$ and $\sigma_{p}>\sigma_{m}$, 157 (4) can be simplified with the approximation:

$$
f_{x o 2}=\frac{\sigma_{p}}{2 \pi} \frac{1}{\varepsilon_{0}} \sqrt{\frac{1}{\left(\varepsilon_{p}-\varepsilon_{m}\right)\left(\varepsilon_{p}+2 \varepsilon_{m}\right)}} .
$$

\section{A. Cellular Culture}

169 Two GBM cell lines were tested in this study, U87-MG and 170 LN18. Both of them derived from malignant stage IV gliomas 171 from adult patients, purchased from the American Type Culture 172 Collection (ATCC). Two conditions were used for the analysis: 173 (i) normal differentiation conditions in DMEM plus Glutamax 174 medium supplemented with 10\% Fetal Bovine Serum (FBS), $1752 \mathrm{mM}$ glutamine and $100 \mathrm{U}$ penicillin $/ 0.1 \mathrm{mg} / \mathrm{mL}$ streptomycin, 176 called NN for "Normal Normoxia Medium", (ii) stringent con177 ditions in selective DMEM/F12 medium, Define Normoxia 178 medium (DN), supplemented with $0.6 \%$ glucose, $1.2 \%$ sodium 179 bicarbonate, $5 \mathrm{mM}$ HEPES, $9.6 \mu \mathrm{g} / \mathrm{mL}$ putrescine, $10 \mu \mathrm{g} / \mathrm{mL}$ 180 ITSS, $0.063 \mu \mathrm{g} / \mathrm{mL}$ progesterone, $2 \mu \mathrm{g} / \mathrm{mL}$ heparin, $20 \mathrm{ng} / \mathrm{mL}$ $181 \mathrm{EGF}, 20 \mathrm{ng} / \mathrm{mL} \mathrm{bFGF}, 1 \mathrm{X}$ penicillin/streptomycin and $2 \% \mathrm{~B} 27$ 182 supplement without vitamin A. Cells are maintained at $37^{\circ} \mathrm{C}$, $1835 \% \mathrm{CO} 2$ in a humidified atmosphere $-95 \%$ air incubator.

184 Actually, under stringent culture conditions, mainly only the 185 most resistant cells with strong aggressiveness special features 186 can survive and grow. As illustrated in Fig. 4, it is hence expected

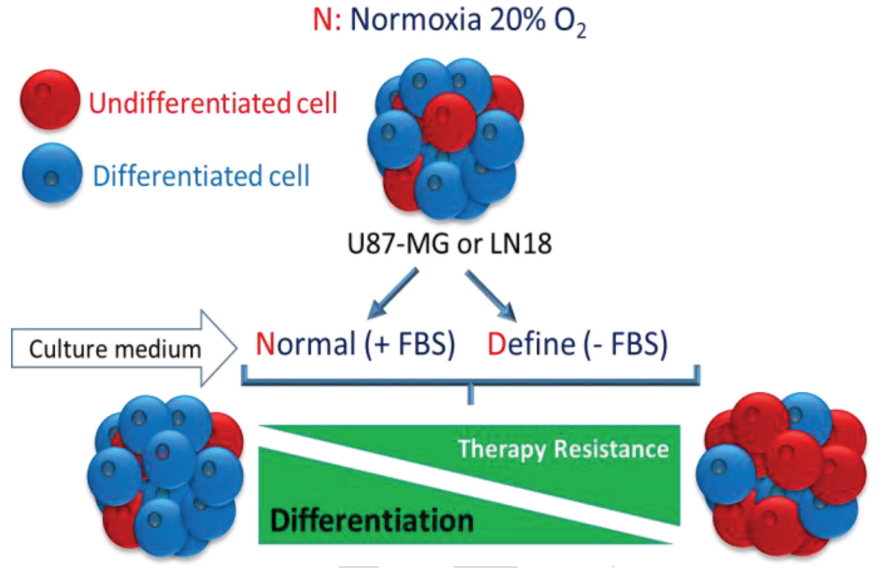

Fig. 4. Representative diagram of the two different culture conditions used for the both GBM cell lines.

to achieve a large enrichment in undifferentiated cells in DN cell 187 cultures.

Finally, after two successive centrifuge washes, cells were 189 suspended in an ion free osmotic medium TRIS buffer-based, 190 composed by a water/sucrose mixture with magnesium chloride 191 ( $\mathrm{pH}$ : 7.4; conductivity: $26 \mathrm{mS} / \mathrm{m}$ ) conventionally used for DEP 192 experiments. The osmolarity value of this DEP medium, mea- 193 sured with a sample of $70 \mu \mathrm{L}$ placed in micro-digital osmometer 194 300-11DR (Type13) varies between 280 and $320 \mathrm{mOSm}$.

\section{B. Tools and Methodology for Cell Crossover}

The main purpose of this study is to characterize GBM cell 198 lines to identify their DEP crossover frequencies in the high fre- 199 quency regime and establish DEP signature according to their 200 different culture conditions (normal culture medium vs. define 201 medium). Each cell population is introduced into the microflu- 202 idic chip, suspended in a DEP medium, by a fluidic inlet driven 203 by a flow controller (Fluigent MFCS) and flows in a Poly- 204 diméthylsiloxane (PDMS) microfluidic channel implemented 205 above dedicated sensors implemented in BiCMOS technology 206 (see Fig. 1). The experiments were done using a $40 \times 40 \mu \mathrm{m} 207$ gap quadrupole electrodes design. This structure is based on 208 four electrodes, set at $90^{\circ}$, combining a pair of thick $(9 \mu \mathrm{m}) 209$ electrodes crossing the microfluidic channel with another pair 210 of thin $(0.45 \mu \mathrm{m})$ electrodes implemented in the middle of the 211 channel [14].

The selected $40 \mu \mathrm{m}$ spacing between each electrodes rep- 213 resent a good compromise between an easy monitoring under 214 microscope of cell motion submitted to both positive or negative 215 DEP forces and the use of moderate RF voltage signal to bias 216 the structure and efficiently act on cells (typically the magnitude 217 of the applied voltage ranges between 2 and $4 \mathrm{~V}_{\mathrm{pp}}$ ). The same 218 frequency adjustable DEP signal has been applied to the left and 219 right electrodes whereas top and bottom ones were grounded. 220 The flow is slowed down, and when a cell arrives near the cen- 221 ter of quadrupole electrodes system, the electrodes were biased 222 with a $500 \mathrm{MHz}$ DEP signal expected to be much higher than 223 the $f_{x o 2}$ and therefore suitable to efficiently trap cells. 


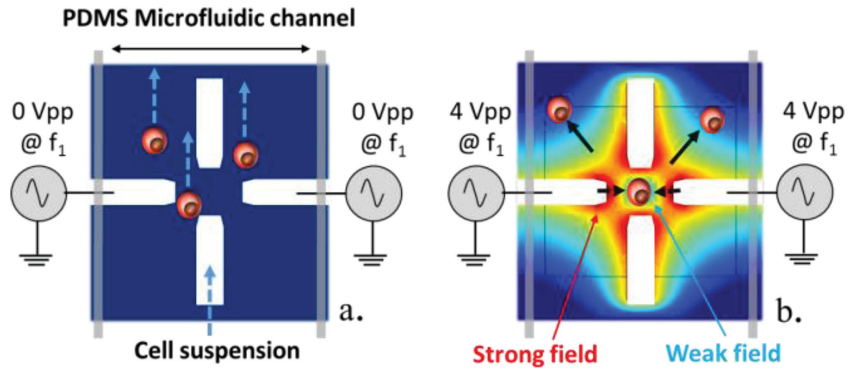

Fig. 5. Cells suspension flowing in the microfluidic system (a). Single cell $\mathrm{nDEP}$ trapping at $500 \mathrm{MHz}$ in the center of the quadrupole electrodes related to the generated electric field (b).

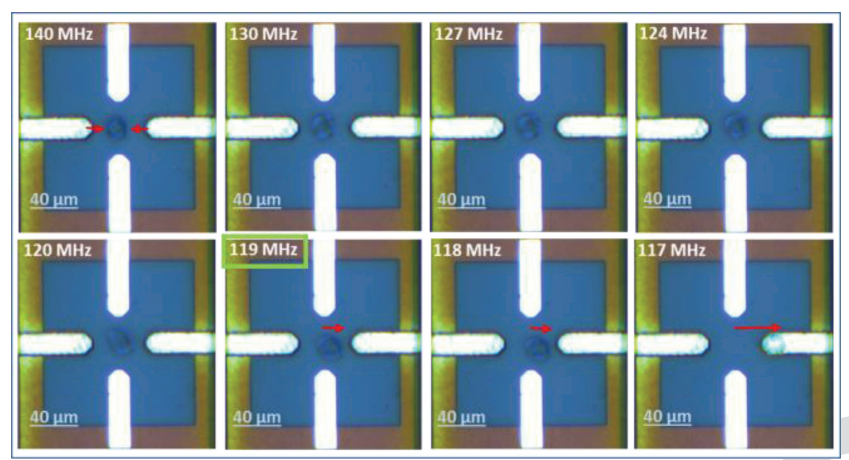

Fig. 6. Cultured in NN - Microscope imaging sequence of GBM cell crossover frequency measurement $(119 \mathrm{MHz})$ by tuning the DEP signal frequency.

At $500 \mathrm{MHz}$, the cell may react in negative DEP and the generated electric field allows individually catching any single cell present near the system center. Indeed, the strong intensity field areas surrounding the center weaker field zone resulted in an electric field cage where the cell could be efficiently trapped (see Fig. 5(b)). The others surrounding cells also reacted in negative DEP and were repelled away the analysis area moving to the outside weaker intensity field zones. Then, the flow was progressively stopped and stabilized (reaching an inlet and outlet pressure equilibrium at each microchannel end). Finally, the cell was only submitted to DEP force and natural gravity. The DEP signal was first turned off for few seconds to check that the investigated cell is no longer subject to other motion forces.

Then, it was turned on again setting the signal frequency above the expected crossover frequency and the characterization could start. To determine the investigated cell crossover frequency, gradual frequencies decreases were applied on the DEP signal: first with fast $10 \mathrm{MHz}$ steps and then once approaching the crossover frequency with $1 \mathrm{MHz}$ ones to refine the measurement. Since the crossover frequency occurred just before the moment when the cell started to be attracted by one of lateral electrodes (switching to positive DEP behavior), a slow $1 \mathrm{MHz}$ step frequency scan allowed to accurately observed this moment. Fig. 6 illustrates an example of trapping and crossover frequency characterization of a GBM cell cultured in normal medium.

Each cell was characterized hence twice or three times and finally released by increasing the inlet channel pressure to renew

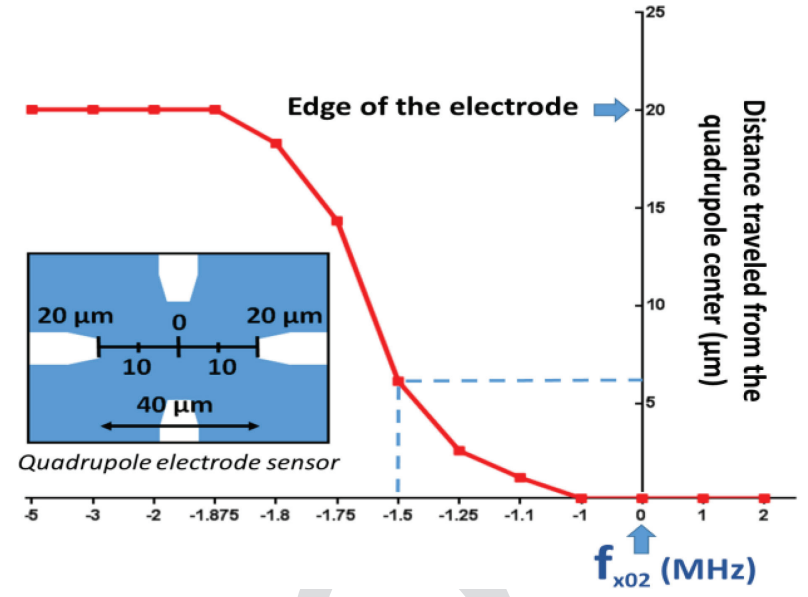

Fig. 7. Simulated cell location change from the center of the quadrupole electrodes system for different DEP signal frequency tuned around the cell crossover frequency.

medium and trap a new cell for characterization following the 253 same approach.

This methodology of crossover frequency measurement has 255 been supported and validated though electro-kinetic transient 256 simulations using COMSOL software. Hence, the displacement 257 of the trapped cell, from the center of quadrupole to the edge 258 of the electrodes, according to the decrease of the DEP signals 259 frequency, can be computed as presented in the graph below. 260

As Fig. 7 shows, the simulations predict that the generated 261 DEP force, once applying a $4 \mathrm{~V}_{\mathrm{pp}}$ DEP signal with a frequency 262 set to $1.5 \mathrm{MHz}$ under the cell crossover frequency, should 263 have a sufficient influence to attract and move it $6 \mu \mathrm{m}$ away 264 from its initial negative DEP trapping location. Actually, such 265 displacement value matches well with the ones observed un- 266 der a microscope during experiments, as illustrated on the Fig. 267 6 photograph taken at $118 \mathrm{MHz}$. Consequently, we can rea- 268 sonably consider that using the proposed cell characterization 269 methodology the cell crossover frequency can be measured with 270 a 1-2 MHz accuracy.

\section{Results, ANALYSIS AND CORRELATION}

\section{A. GBM Cell Lines Phenotypic Profiles}

First, control experiments were carried out to confirm the en- 274 richment of undifferentiated cell population in define medium. 275 Comparative analysis of the gene expression (mRNA levels) 276 of the stemness lineage was assessed in the cells cultured 6277 days in normal culture medium vs. define medium (see Fig. 8). 278 Analyzed CSCs markers (Oct-4, Sox2 and Nanog) showed an 279 overexpression in cells cultured in define medium (in red) com- 280 pared to those cultured in normal medium (in blue).

281

These results were completed by analyzing protein levels with 282 flow cytometry. They showed an enhancement of undifferenti- 283 ated markers expression in both U87-MG (Fig. 9(a) and (b)) and 284 LN18 (see Fig. 9(c) and (d)) cell lines cultured in define medium 285 compared to normal medium culture. These biological results 286 validate the enrichment of CSC by culture in define medium. $\quad 287$ 

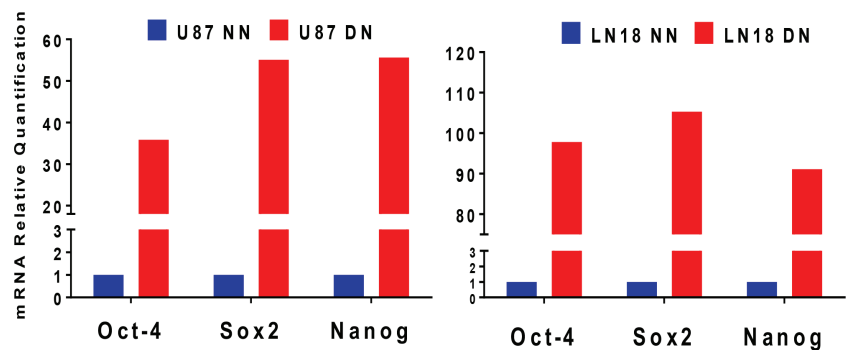

Fig. 8. Comparative analysis of gene expression of three undifferentiated markers: Oct-4, Sox 2 and Nanog, in U87-MG and LN18 cell lines, cultured 6 days in normal medium (NN: blue histograms) or in define medium (DN: red histograms), measured by Real Time PCR (Polymerase Chain Reaction).
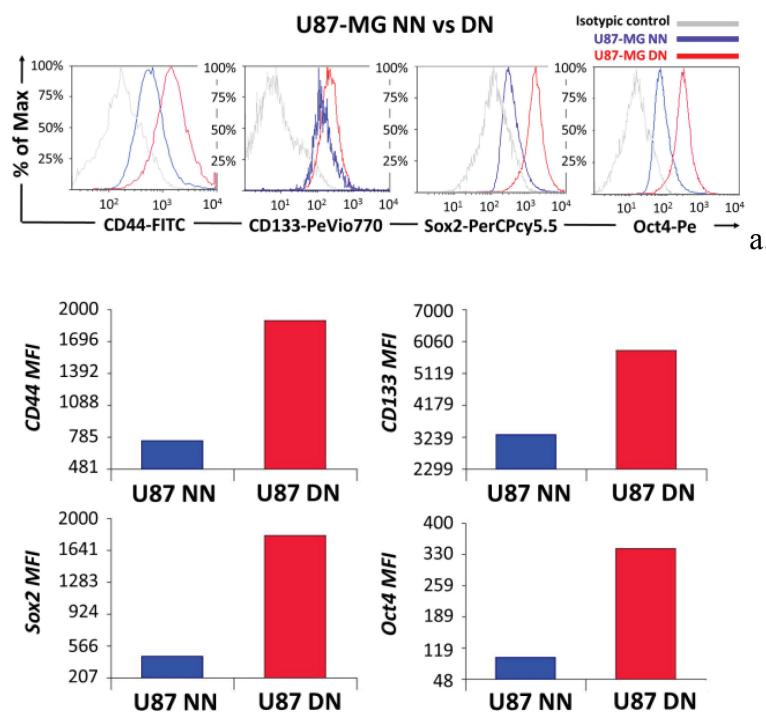

a.
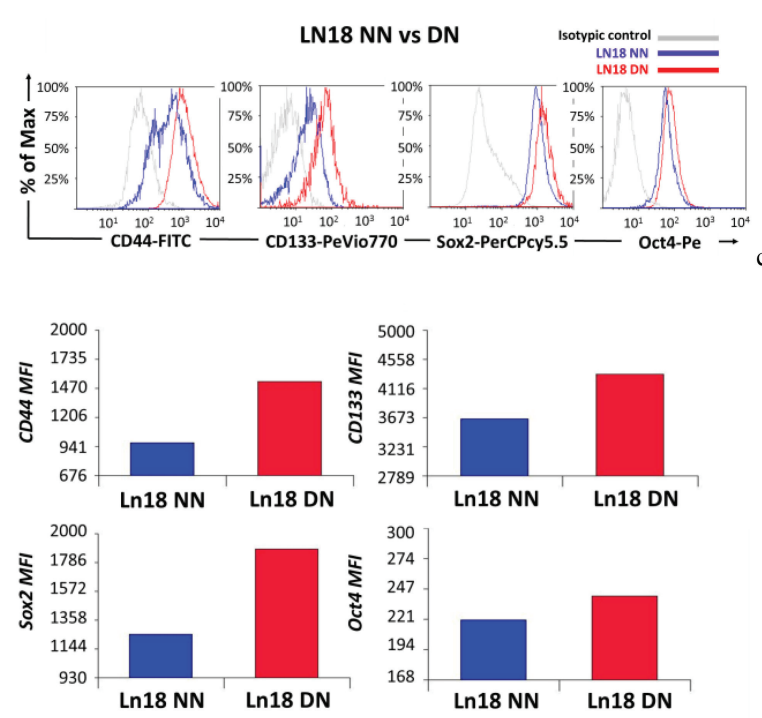

Fig. 9. Comparative analysis of the undifferentiation markers expression CD44, CD133, Oct-4, Sox2 in both U87-MG and Ln18 cell lines, cultured 6 days in normal medium (NN: blue histograms) or in define medium (DN: red histograms), analyzed by multi-parameteric flow cytometry (BD Fortessa). (a) and (c) graphs represent percentage of expression and (b) and (d) histograms represent the mean fluorescent intensity (MFI) for each marker expression in U87-MG and LN18 respectively, grey graphs show the isotypes (unlabeled) negative control condition.

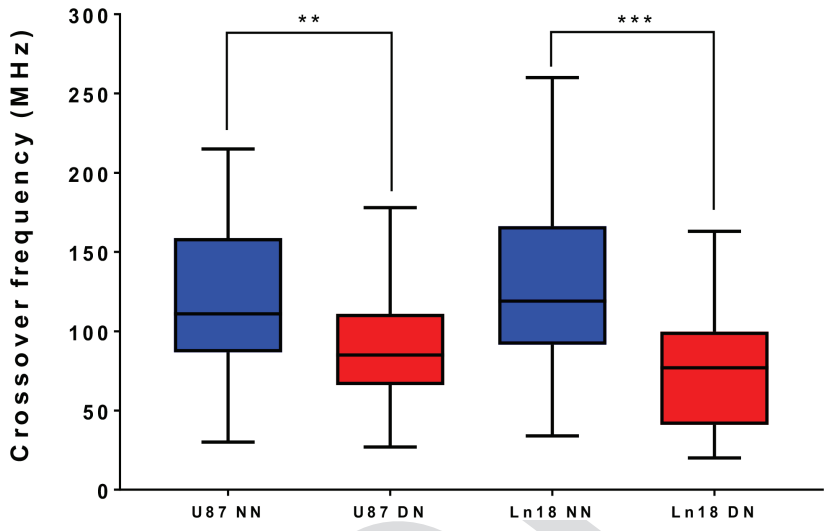

Fig. 10. Graphic box plots representation of U87-MG and LN18 cells crossover frequencies, cultured in two different conditions: normal medium (NN) and define medium (DN). The p value was determined using One-way ANOVA test. ${ }^{* * *}$ represents $p$ value $<.0001,{ }^{* *}$ represents $p$ value $<.001$.

TABLE I

SUMMARY OF CROSSOVER FREQUENCY MEASUREMENTS (MHz)

\begin{tabular}{|c|c|c|c|c|c|c|c|}
\hline $\begin{array}{c}\text { Cell } \\
\text { lines }\end{array}$ & $\begin{array}{c}\text { Number } \\
\text { of cells }\end{array}$ & Avg & Median & $\begin{array}{c}\text { Dev } \\
\text { Std }\end{array}$ & SEM & Min & Max \\
\hline $\begin{array}{c}\text { U87 } \\
\text { NN }\end{array}$ & 104 & 120 & 111 & 45.11 & 4.36 & 30 & 215 \\
\hline $\begin{array}{c}\text { U87 } \\
\text { DN }\end{array}$ & 57 & 91 & 85 & 36.34 & 4.73 & 27 & 178 \\
\hline $\begin{array}{c}\text { Ln18 } \\
\text { NN }\end{array}$ & 138 & 128 & 119 & 53.11 & 4.47 & 34 & 260 \\
\hline $\begin{array}{c}\text { Ln18 } \\
\text { DN }\end{array}$ & 116 & $\mathbf{7 6}$ & 77 & 34.47 & 3.17 & 20 & 163 \\
\hline
\end{tabular}

\section{B. GBM Cell Crossover Frequencies}

In order to support these results obtained at the biological 289 level, the two GBM cell lines were characterized by establish- 290 ing their UHF-DEP crossover frequencies according to their 291 different culture conditions.

The set of crossover frequencies measured for both cell lines 293 (U87-MG and LN18) cultured in the two different conditions 294 (normal culture medium vs. define medium) is represented in 295 the Fig. 10 (one should notice that the middle bar here represents 296 the median value of the whole collected data). The considered 297 crossover frequency corresponds to the frequency for which the 298 trapped cell just starts to move away from the center of electrodes 299 quadrupole. Based on statistical analysis of the results observed 300 in the four populations, culturing cells in a define environment 301 seems to have a real impact on the measured crossover frequen- 302 cies, according to GBM intracellular characteristics changes. 303 The set of statistics concerning the characterization of U87-MG 304 and LN18 cells crossover frequencies is summarized in Table I, 305 listing the average, median, standard deviation, standard error, 306 minimum and maximum crossover frequency values for each 307 cell population. 308

As shown a significant number of cells have been charac- 309 terized showing statistically consolidated data. The large stan- 310 dard deviation, and error standard, observed for U87-MG and 311 LN18 NN cell pool can be explained by the natural cell line 312 
313 heterogeneity, including a large number of different differenti314 ated cells but also some few undifferentiated occurrence in the 315 pool. On the other hand, the DN cell pools may concentrate 316 a much higher number of undifferentiated and low differenti317 ated cells; since DN culture conditions are not favorable for 318 differentiated cell growth.

319 Considering the measured crossover frequencies, these two 320 GBM cell lines exhibit the same behavior according to the two 321 different culture conditions. Actually, the undifferentiated en322 riched populations (DN) show much lower crossover frequen323 cies than the cells cultured in normal conditions, although some 324 crossover frequencies overlap exist between these two popu325 lations - U87-MG: Average of $120 \mathrm{MHz}$ for NN vs. $91 \mathrm{MHz}$ 326 for DN - LN18: Average of $128 \mathrm{MHz}$ for NN vs. $76 \mathrm{MHz}$ 327 for DN. This decrease demonstrates a significant difference (il328 lustrated by the run ANOVA statistical analysis tests resulting 329 in a very low $p$ value) between these two population profiles 330 obtained by different culture conditions. This finding proves a 331 real difference on the intracellular dielectric characteristics of 332 the undifferentiated cells enriched populations compared to dif333 ferentiated cells, reflecting the intrinsec biological properties 334 differences.

335 This difference outlines a great potential for discrimination 336 of cell subpopulations within the whole tumor mass. Therefore 337 such technique is highly promising to achieve discrimination 338 and even isolation of undifferentiated cells allowing potential 339 cell sorting of these undifferentiated subpopulations related to 340 the CSC subpopulation.

\section{CONCLUSION}

342 We described here a novel method of cellular subpopula343 tions' discrimination, which completes the classical biological 344 approaches, based on the differential expression levels of a set 345 of markers. These populations, with different cellular differen346 tiation status, are discriminated using real time measurement 347 on microfluidic lab-on-chip (LOC) platform implemented in 348 CMOS technology. Both selected GBM cell lines, showed a 349 strong correlation between the biological markers differences 350 and the measured DEP frequency signatures according to the 351 different culture conditions. Differences on crossover frequen352 cies obtained for each subpopulation, showed a great discrimina353 tion potential especially for the development of a novel method 354 to characterize Cancer Stem Cells. Thus, we confirmed the bi355 ological differences analyzed by routine methods, using DEP 356 signatures differences, which complete the characterization of 357 stemness properties cells. These results correlate to the bio358 logical differences at the functional level. The undifferentiated 359 properties of CSCs are associated to intracellular changes and re360 flecting their high aggressiveness potential. This technic allows 361 screening of a new cell discrimination parameter, the intracel362 lular differences and physical properties of cells, without any 363 labeling, without affecting cell integrity and viability. Hence, 364 based on the UHF-DEP spectroscopy method, we detected and 365 characterized the undifferentiated cells with unique capabilities 366 to screen biological specificities by investigating the intracellu367 lar dielectric properties.
Finally, this method confirms a high potential of emerging 368 lab-on-chip (LOC) platforms in the diagnosis and the treatment 369 of glioblastoma.

\section{REFERENCES}

[1] M. Cheray et al., "Cancer stem-like cells in glioblastoma," De 372 Vleeschouwer S1, editor. Glioblastoma [Internet]. Brisbane, AU: Codon, 373 Sep. 2017, Ch. 4.

[2] J. Chen, R. M. McKay, and L. F. Parada, "Malignant glioma: lessons from 375 genomics, mouse models, and stem cells," Cell, vol. 149, no. 1, pp. 36-47, 376 30 Mar. 2012. doi: 10.1016/j.cell.2012.03.009.

[3] A. Bradshaw, A. Wickremsekera, S. T. Tan, L. Peng, P. F. Davis, and 378 T. Itinteang, "Cancer stem cell hierarchy in glioblastoma multiforme," 379 Front Surg, vol. 3, p. 21, 15 Apr. 2016. doi: 10.3389/fsurg.2016.00021. 380 eCollection 2016.

[4] F. Zeppernick, R. Ahmadi, B. Campos, C. Dictus, B. M. Helmke, 382 N. Becker, P. Lichter, A. Unterberg, B. Radlwimmer, C. C. Herold- 383 Mende, "Stem cell marker CD133 affects clinical outcome in glioma 384 patients," Clin Cancer Res., vol. 14, no. 1, pp. 123-9, 1 Jan. 2008. doi: 385 10.1158/1078-0432.CCR-07-0932. 386

[5] Q. Liu, C. Wu, H. Cai, N. Hu, J. Zhou, and P. Wang, "Cell-based biosensors 387 and their application in biomedicine." Chem. Rev., vol. 114, 6423-6461, 388 2014.

[6] N. Abd Rahman, F. Ibrahim, and B. Yafouz, "Dielectrophoresis for 390 biomedical sciences applications: A review," Sensors, vol. 17, p. 449, 391 2017.

[7] R. Pethig, "Dielectrophoresis: Theory, methodology and biological 393 applications." New York, NY, USA: Wiley, Mar. 25, 2017, doi: 394 10.1002/9781118671443.

[8] P. R. C. Gascoyne and J. V. Vykoukal, "Dielectrophoresis-based sam- 396 ple handling in general-purpose programmable diagnostic instruments," 397 in Proc. IEEE Inst. Electr. Electron. Eng., vol. 92, no. 1, pp. 22-42,1 398 Jan. 2004. doi: 10.1109/JPROC.2003.820535.

[9] E. Salimi, K. Braash, M. Butler, D. J. Thomson, and G. E. Bridges, 400 "Dielectric model for Chinese hamster ovary cells obtained by dielec- 401 trophoresis cytometry," Biomicrofluidics, vol. 10, p. 014111, 2016. doi: 402 10.1063/1.4940432. microwave biosensors," Sensors Actuators: A. Physical, vol. 216, no. 1, 405 pp. 405-416, Sep. 2014.

[11] F. Artis et al., "Microwaving biological cells: intracellular analysis with 407 microwave dielectric spectroscopy," IEEE Microw. Mag., vol. 16, no. 4, 408 pp. 87-96, May 2015.

[12] X. Ma, X. Du, H. Li, X. Cheng, and J. C. M. Hwang, "Ultra-wideband 410 impedance spectroscopy of a live biological cell," in IEEE Trans. Microw. 411 Theory Techn., to be published, doi: 10.1109/TMTT.2018.2851251. 412

[13] H. A. Pohl, "The motion and precipitation of suspensoids in divergent 413 electric fields," J. Appl. Phys., vol. 22, 869-871, 1951.

[14] F. Hjeij et al., "UHF dielectrophoretic handling of individual biological 415 cells using BiCMOS microfluidic RF-sensors," in Proc. 46th Eur. Microw. 416 Conf. (EuMC), London, Oct. 2016.

[15] G. S. Fiorini and D. T. Chiu, "Disposable microfluidic devices: Fabrica- 418 tion, function, and application," Bio. Techn., vol. 38, pp. 429-446, 2005. 419

[16] L. M. Broche, F. H. Labeed, and M. P. Hughes, "Extraction of dielec- 420 tric properties of multiple populations from dielectrophoretic collection 421 spectrum data," Phys. Med. Biol., vol. 50, pp. 2267-2274, 2005.

[17] X. Du, X. Ma, H. Li, Y. Ning, X. Cheng, and J. C. M. Hwang, "Validation 423 of clausius-mossotti function in single-cell dielectrophoresis," in Proc. 424 IEEE/MTT-S Int. Microw. Biomed. Conf., 2018.

[18] K. Asami, Y. Takahashi, and S. Takashima, "Dielectric properties of mouse 426 lymphocytes and erythrocytes," Biochimica et Biophysica Acta, vol. 1010, 427 pp. 49-55, 1989.

[19] S. Afshar, A. Fazelkhah, E. Salimi, M. Butler, D. Thomson, and G. Bridges, 429 "Change in the dielectric response of single cells induced by nutrient de- 430 privation over a wide frequency range," in Proc. IEEE MTT-S Int. Microw. 431 Symp. (IMS).

[20] J. Gimsa, P. Marszalek, U. Loewe, and T. Y. Tsong, "Dielectrophorsis and 433 electrorotation of neurospora slime and murine myeloma cells," Biophys., 434 J., vol. 60, pp. 749-760, 1991.

[21] C. Chung, R. Pethig, S. Smith, and M. Waterfall, "Intracellular potas- 436 sium under osmotic stress determines the dielectrophoresis cross-over 437 frequency of murine myeloma cells in the MHz range," Electrophoresis, 438 vol. 39, pp. 989-997, 2018. 

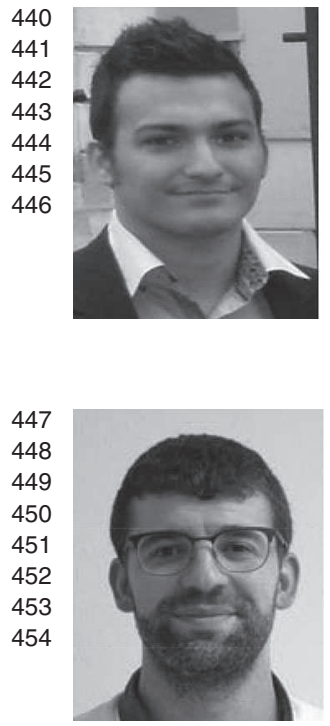

Rémi Manczak received the Ph.D. degree in physics and material sciences from the University of Toulouse, Toulouse, France, in 2016. Since 2017, he has been working as a Postdoctoral Researcher Associate in the SUMCASTEC project with the University of Limoges, Limoges, France.

Sofiane Saada received the Ph.D. degree in Immunology and Oncology from the University of Limoges, France, in 2015. He was a postdoctoral associate at the University of Pittsburgh Medical Center (UPMC) (USA) (2015-2017). In 2017, he came back to University of Limoges, where he is working as a postdoctoral associate in the SUMCASTEC project.

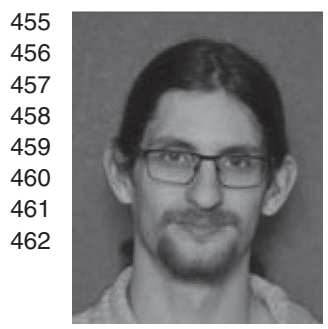

Thomas Provent has been working toward the Ph.D degree with the University of Limoges, France, since 2017. His research is linked directly with the SUMCASTEC project with the XLIM Research Institute on the topic of the development and characterization of a sensor to isolate and characterize cancer stem cells.

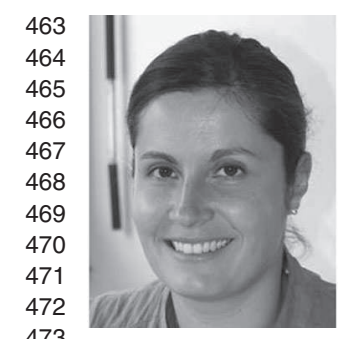

Claire Dalmay received the Ph.D. degree in electrical engineering from the University of Limoges, Limoges, France, in 2009. She holds a post-doctoral position with the Centre National de la Recherche Scientifique (CNRS), SATIE-BIOMIS, IFR d'Alembert, ENS Cachan, working on the development of microfluidic biochips for cell electroporation and cell handling. She is currently an assistant professor at the University of Limoges XLIM Laboratory. Her research interests are focused on microsystems for biomedical applications and additive microfabrica474 tion technologies for RF and microwave components.

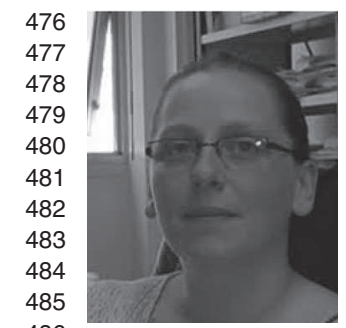

Barbara Bessette received the Ph.D. degree in neuroscience and oncology from the University of Limoges, France, in 2006. She worked for one year in Paris, France, on pediatric brain tumors and the characterization of cancer stem cells in these tumors. She followed her post-doctoral experience by collaborating and working for 3 years on the GLIADYS project with IDD-Biotech (International Drug Development Biotech), specializing in monoclonal antibodies production in Lyon, France. She is currently a full-time assistant Professor at the University of Limoges in 487 the Department of Physiology, and she leads research with the CAPtUR team 488 Her current research activity focuses on cancer stem cells in glioblastoma and 489 the role of neuropeptides in their therapeutic resistance capacity. As one of 490 the workpackages leaders in SUMCATEC (H2020 European Project), she par491 ticipates in work to determine cancer stem cell electromagnetic signature in 492 glioblastoma and medulloblastoma.

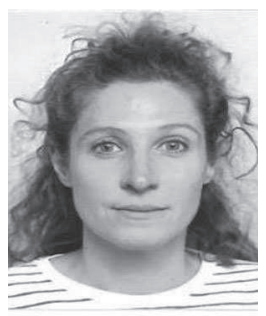

Gaëlle Bégaud is a pharmacist who received the M.S. 494 degree in molecular, cellular, and integrated phys- 495 iopathology from the University of Toulouse, France, 496 in 2003 and the Ph.D. degree in analytical chemistry 497 and cellular biology from the University of Limoges, 498 France, in 2007. In 2008, she joined Limoges Uni- 499 versity, where she is now an assistant professor in 500 analytical chemistry and a researcher in the EA3842, 501 CAPTuR team. Her research activity linked to the 502 SUMCASTEC project is centered on the optimizaton 503 of the use of Sedimentation Field Flow Fractionation 504 (SdFFF) to sort Brain Cancer Stem Cells (BTSC) from complex populations 505 such as neuroblastoma or glioblastoma cell lines. Her research interests include 506 the implementation of new SdFFF prototypes for biological applications. $\quad 507$

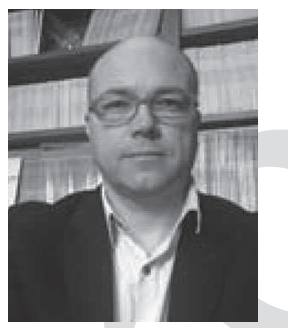

Serge Battu is a pharmacist who received the M.S. 509 degree in Chemistry from the University of Toulouse, 510 France, in 1993, and the Ph.D. degree in Cellular and 511 Molecular Biology from the University of Limoges, 512 France, in 1997. In 1998 he joined Limoges Univer- 513 sity, where he is now a full professor in Analytical 514 Chemistry and instrumentation and a researcher in 515 the EA3842 team. His research research activity is 516 centred on development of news SdFFF prototypes 517 and to use SdFFF to sort cancer stem cells (BTSC) 518 from complex populations such as neuroblastoma or 519 glioblastoma cell line. 520

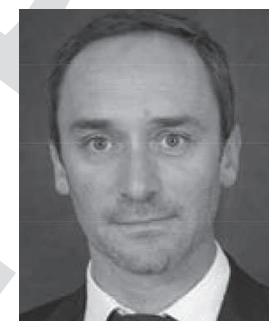

Pierre Blondy received the Ph.D. and Habilitation 522 degrees from the University of Limoges, Limoges, 523 France, in 1998 and 2003, respectively. He joined 524 the Centre National de la Recherche Scientifique as 525 a research engineer in 1998 and the University of 526 Limoges in 2006, where he is currently a Professor. 527 From 2011 to 2015, he held an Institut Universitaire 528 de France research chair. He was a visiting researcher 529 at the University of Michigan, Ann Arbor, USA, in 530 1997 and at the University of California at San Diego, 531 La Jolla, USA, in 2006 and 2008. He founded a re- 532 search group in 2003 at XLIM on innovative technologies for microwave and 533 millimeter-wave applications, and he was involved in many research projects, 534 funded by national and european public agencies and companies. He has been 535 working extensively on tunable filters, RF-MEMS components, and reliability. 536 His group is also studying the properties of phase change transition materials 537 and their applications to microwaves. Switches, power limiters, and tunable 538 filters have been developped using innovative materials like Vanadium Dioxide 539 and Germanium Telluride. Dr. Blondy was an Associate Editor for the IEEE 540 MicROWAVE AND WIRELESS COMPONENTS LETTERS in 2006. He has been a 541 member of the IEEE International Microwave Conference Technical Program 542 Committee since 2003, and he is also serving on the technical program of the 543 European Microwave Conference. He is the past chair of the MTT-S Technical 544 Committee 21 on RF-MEMS.

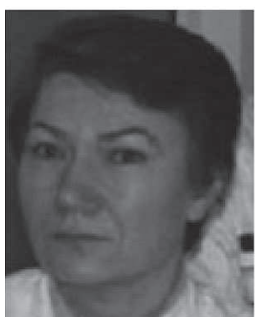

Marie-Odile Jauberteau received the Medical Doc- 547 tor degree from Limoges University and the Ph.D. 548 degree from the University of Paris VI. She has been 549 a Medical professor of Immunology since 2000 at 550 Limoges University, Faculty of Medicine. She is the 551 director of the Research Team EA 3842, Control of 552 Cell Activation, Tumor Progression and Therapeutic 553 Resistance (CAPTuR), created in 2004. Her research 554 is focused on oncology, especially mechanisms of 555 cell survival and cancer stem cells. 

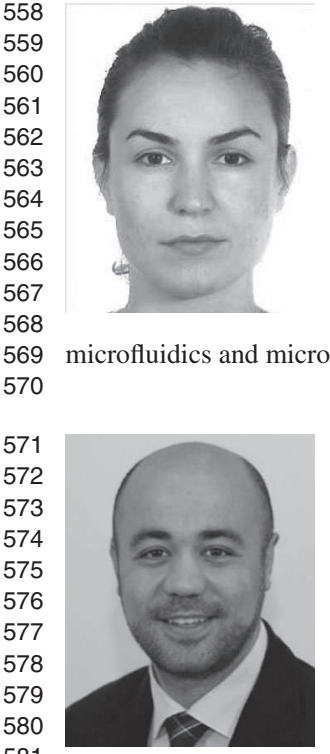

Canan Baristiran Kaynak received the B.S degree from the Chemistry department of Istanbul Technical University and the master degree in Material Science and Engineering at Sabanci University, Istanbul, Turkey, in 2007. She received the Dr.-Ing. degree from the institute of high frequency and semiconductor system technologies from Technical University of Berlin, Germany, in 2012. Since 2008, she has held a position as scientist in the Technology Department at IHP Microelectronics. She is leading projects including heterogeneous integration of silicon-based microfluidics and microbolometer devices into BiCMOS technology.

Mehmet Kaynak received the B.S degree from the Electronics and Communication Engineering Department of Istanbul Technical University (ITU) in 2004, the M.S degree from the Microelectronic program of Sabanci University, Istanbul, Turkey in 2006, and the Ph.D. degree from Technical University of Berlin, Berlin, Germany, in 2014. He joined the technology group of IHP Microelectronics, Frankfurt (Oder), Germany, in 2008. From 2008 to 2015, he led the MEMS development at IHP. Since 2015, he has been the department head of Technology at 582 IHP. Dr. Kaynak is being affiliated as Adjunct Professor at Sabanci University, 583 Turkey.

584

585

586

587

588

589

590

591

592

593

594

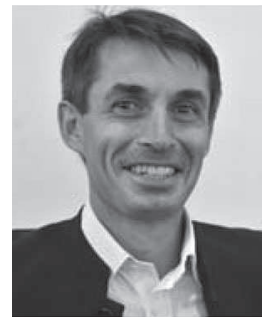

Fabrice Lalloué received the Ph.D. degree in neuro- 599 biology and neurodevelopment from the University 600 of Limoges, France, in 2001. He is currently Profes- 601 sor for the University of Limoges in the Department 602 of Physiology and Neurosciences (Neuro-Oncology). 603 $\mathrm{He}$ is also assistant director of the research team 604 EA3842 CAPTuR (Control of cell Activation, Pro- 605 gression of Tumor and therapeutic Resistance) at 606 the Faculty of Medicine. In 2000, he was visiting 607 the Institute of Development, Aging and Cancer in 608 Sendai, Japan. His research activities currently ad- 609 dress Neuro-Oncology, Glioblastoma, Brain Tumour, Initiating stem cells, adult 610 neural stem cells, and Tumour Biomarkers. He is involved in the Scientific Pilot 611 Committee of the Canceropole Grand Sud-Ouest consortium. He has authored 612 or co-authored over 50 publications in research articles and conferences and 613 holds seven patents.
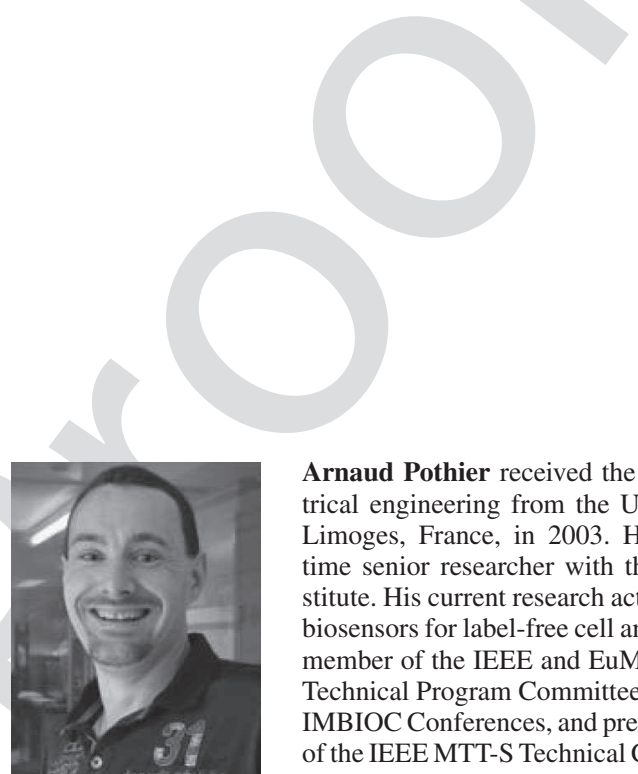

Arnaud Pothier received the Ph.D. degree in elec- 616 trical engineering from the University of Limoges, 617 Limoges, France, in 2003. He is currently a full- 618 time senior researcher with the XLIM research in- 619 stitute. His current research activity is focused on RF 620 biosensors for label-free cell analysis. Dr. Pothier is a 621 member of the IEEE and EuMA society, member of 622 Technical Program Committees of IMS, EuMW, and 623 IMBIOC Conferences, and previously served as chair 624 of the IEEE MTT-S Technical Committee MTT-10 on 625 Biological Effect and Medical Applications. $\quad 626$ minimally invasive animal telemetry, and biomedical technology, along with ergy harvesting and RF-MEMS for reconfigurable antenna arrays.

Cristiano Palego received the M.S. degree in Electri-
cal Engineering from the University of Perugia, Italy, in 2003 and the Ph.D. degree in Microwave Engineerges, France, in 2007. He was a postdoctoral associate then a research scientist at Lehigh University, Bethlehem, PA, USA, (2007-2017). In 2013, he joined Bangor University, U.K., where he is now an associate professor in smart sensors and instrumentation and a researcher in the Medical Microwave Systems group. His research interests include micro/nanotechnology, RF-MEMS for reconfigurable antenna arrays. 